\title{
Dense Graphene Nanoplatelet / Yttria Tetragonal Zirconia Composites: Processing, Hardness and Electrical Conductivity
}

\author{
Angela Gallardo-López a , , Iván Márquez-Abril a, Ana Morales-Rodríguez a, Antonio \\ Muñoz ${ }^{\text {a }}$, Rosalía Poyato ${ }^{b}$ \\ ${ }^{a}$ Dpto. de Física de la Materia Condensada, ICMSE, CSIC-Universidad de Sevilla, apdo. 1065, 41080 Sevilla, Spain \\ ${ }^{b}$ Instituto de Ciencia de Materiales, ICMSE, CSIC-Universidad de Sevilla, Avda. Américo Vespucio 49, 41092 Sevilla, Spain
}

Elsevier use only: Received date here; revised date here; accepted date here

\begin{abstract}
Yttria tetragonal zirconia ceramic composites with 1, 2.5, 5 and 10 vol\% nominal contents of graphene nanoplatelets (GNPs) were fabricated and characterized. First, the GNP dispersion in isopropanol was optimized to de-agglomerate the GNPs without damaging their structure. Then, submicrometric fully dense composites were obtained via spark plasma sintering (SPS) at $1250^{\circ} \mathrm{C}$ with a $5 \mathrm{~min}$ holding time. The processing routine produced a nearly homogeneous GNP dispersion in the ceramic matrix, and the GNPs preferential orientation was perpendicular to the sintering compression axis. The Ceramic grain refinement due to the GNPs was also detected. The Vickers hardness measured on the plane perpendicular to the sintering compression axis (basal plane) was lower than on the cross sections. This anisotropy increased with the increasing GNP content, while the average hardness decreased. The electrical conductivity was also highly anisotropic, up to seven times higher for the basal planes. The electrical percolation threshold for these composites was estimated to be between 2.2 and 4.4 vol\% of the GNP measured content. (C) 2001 Elsevier Science. All rights reserved
\end{abstract}

Keywords: Ceramic composites; 3YTZP; graphene nanoplatelets; processing; microstructure.

\section{Introduction}

Amongst advanced ceramics, $3 \mathrm{~mol} \%$ yttria tetragonal zirconia polycrystals (3YTZP) stand out because of their hardness, high Young's modulus, chemical stability, low friction coefficient, high wear resistance, ionic conductivity, mechanical strength and high fracture toughness. This last attribute is due to the tetragonal-monoclinic martensitic transformation toughening, which impedes fracture propagation [1]. The 3YTZP is used as a biomaterial and in hip and knee joint replacements as a result of these excellent properties together with its high biocompatibility and low density [2].

The addition of carbon nanostructures in ceramics has become an interesting research topic due to the enhancement of the mechanical, thermal and electrical properties of the resulting carbon nanostructure/ceramic composites [3]. Since carbon is one of the most abundant materials on earth, these composites are an inherently sustainable and economical technology [4]. The two-dimensional character of graphene makes it a challenging candidate for engineering new materials because it can improve the interfacial contact with different phases [5]. The initial studies showed that graphene

\footnotetext{
${ }^{*}$ Corresponding author. Tel.: +0-000-000-0000 ; fax: +0-000-000-0000 ; e-mail: author@ institute.xxx .
} 
nanoplatelets (GNPs) (also called graphite nanoplatelets) could be used as a viable and inexpensive filler substitute for carbon nanotubes (CNTs) and were carried out on polymers [6]. This thesis was supported by the excellent in-plane mechanical, structural, thermal and electrical properties of graphite [7]. The graphene hexagonal structure is very stable due to strong in-plane bonds, while the different graphene layers in GNPs can easily delaminate into individual graphene sheets by applying mechanical stress due to the weak pz bonding between the layers [8]. This allows graphene to be produced either by the top-down exfoliation of graphite stacks [9] or by the bottom-up assembly of smaller atoms and molecules [10,11]. As an advantage over carbon nanotubes, graphene can be produced at the ton scale without high temperature and metal catalysts, allowing relatively cheap production for large scale applications [12]. Graphene/ceramic composites have further advantages over carbon nanotube composite ceramics, such as the less restrictive processing conditions [4].

Pristine, single layered graphene has outstanding electrical, thermal and mechanical properties [8], including a high electron mobility at room temperature $\left(2.5 \times 105 \mathrm{~cm}^{2} \mathrm{~V}^{-1} \mathrm{~s}^{-1}\right)$ [13] exceptional thermal conductivity $\left(5000 \mathrm{~W} \mathrm{~m}^{-1} \mathrm{~K}^{-1}\right)$ [14] and superior mechanical properties with a Young's modulus of $1 \mathrm{TPa}[15]$ due to the $\mathrm{sp} 2$ hybridization. Therefore, graphene may greatly enhance the electrical conductivity of composites when added to an insulating ceramic matrix. The possibility of precise micromachining of electrically conductive hard ceramic composites could be exploited in the fabrication of different devices, such as Micro Electro-Mechanical Systems (MEMS), for high temperature applications [16]. Although most of the extraordinary properties of graphene nanosheets are only associated with individual sheets, GNPs formed by the stacking of approximately 10 to 100 sheets, with a 5-10 $\mu \mathrm{m}$ planar dimension and a $10-100 \mathrm{~nm}$ thickness, also exhibit outstanding properties and are much less expensive alternatives, since achieving complete and homogeneous dispersion of individual graphene sheets in various solvents is a significant challenge. The high surface area makes graphene prone to form irreversible agglomerates [17] or to even restack to form graphite through Van der Waals interactions [8]. Several key issues must be addressed to obtain high performance graphene/ceramic composites, including the homogeneous dispersion of graphene with minimal restacking, the effective mixing with the ceramic and the understanding the interfacial structure and properties.

The limited existing studies on graphene/zirconia composites mostly used reduced graphene oxide (rGO), smaller and thinner than the GNPs used in this study, and point to a noticeable increase in the fracture toughness and electrical conductivity of the ceramic matrix $[18,19]$. An electrical conductivity of $\sim 104 \mathrm{~S} / \mathrm{m}$ was obtained for SPSed 3YTZP composites with a 4.1 vol\% rGO (7-8 nm thick) by Shin \& Hong [18], while the percolation threshold was estimated at $2.5 \mathrm{vol} \% \mathrm{rGO}$

Regarding the mechanical properties, the hardness of rGO/3YTZP composites decreased gradually with the increasing rGO content, while the fracture toughness increased $34 \%$ with respect to the monolithic 3YTZP for composites with $4.1 \mathrm{vol} \%$ rGO [18]. This increase in the fracture toughness is significantly larger than that of zirconia composites with similar percentage of carbon nanotubes (CNT). Some authors have used pressureless sintering (PLS) to produce graphene/zirconia composites. Yin et al. [20] used PLS in Ar to fabricate $\mathrm{rGO} / 3 \mathrm{YTZP}$ composites with a high graphene content from GO, and obtained a fine microstructure with pores due to the GO pyrolization. Ramesh et al. [21] used PLS in air to fabricate GO/3YTZP composites and obtained the enhanced densification, hardness and fracture toughness with minimal GO additions $(<1 \mathrm{wt} \%)$. The PLS is a simple sintering method that results in composites that exhibit lower anisotropy and lower density than those sintered by methods involving uniaxial pressures. The study of graphene/3YTZP composites is also challenging because graphene may influence the tetragonal to monoclinic and cubic phase ratios in the matrix [22], which could affect the fracture toughness. However, reports indicate the negligible effect of GO on the tetragonal to monoclinic transformation by low temperature degradation for GO/3YTZP composites [21].

Minimal studies have been published concerning 3 YTZP reinforcement with GNPs. Chen et al. [23] reported a $61 \%$ increase in the fracture toughness in 
GNP/3YTZP SPSed composites with a very low GNP content $(0.01 \mathrm{wt} \%)$. The GNPs used in their study were $0.5-2 \square \mathrm{m}$ in diameter and 0.8-1.2 nm thick, so they could instead be considered as few layered graphene (FLG). The use of such a small GNP content may be sufficient to benefit the matrix reinforcement, but it does not affect the electrical conductivity of the composites. The problem with using a higher GNP loading is aggregation, necessitating research on the homogeneous dispersion of graphene during processing. There is a recent study on a GNP/3YTZP system $(15-25 \mu \mathrm{m}$ diameter, 6-8 $\mathrm{nm}$ thickness GNPs) by Markandan et al. [24] who used PLS to obtain composites with electrical conductivity of $0.28104 \mathrm{~S} / \mathrm{m}$ with $2 \mathrm{wt} \%$ GNP.

In this work, the fabrication of submicrometric fully dense and highly anisotropic conductive composites with different competitive GNP contents and homogenous GNP distribution in a 3YTZP ceramic matrix is pursued. Wet powder mixing and spark plasma sintering was used as the processing and sintering techniques. Before mixing, optimization of the GNP de-agglomeration was also performed. The presence of tetragonal, monoclinic and cubic phases in the ceramic matrix was determined by Xray diffraction, And the GNP structural integrity was evaluated by Raman spectroscopy after deagglomeration treatments both before and after sintering the composites. Distribution of GNPs into the ceramic matrix was assessed by microstructural characterization of the composites via scanning electron microscopy (SEM). The influence of the GNP content on the composites' hardness and on their electrical conductivity has also been evaluated and compared to a reference monolithic 3YTZP sample sintered under the same conditions.

\section{Experimental}

\subsection{Processing and sintering}

\subsubsection{GNPs de-agglomeration}

GNPs with $\leq 5 \mu \mathrm{m}$ planar diameter and 50-100 nm thicknesses were supplied by Angstron Materials
(Dayton, Ohio, EEUU). The GNPs can form agglomerates [17] which complicate their dispersion in the ceramic matrix, and instead producing an inhomogeneous distribution in the final sintered composite. Therefore, different treatments prior to sintering have been applied to different GNP suspensions to determine the most effective method to avoid these agglomerates. One method is the ultrasonic agitation of the GNP suspensions in isopropanol with different energies and for different periods of time. Isopropanol was chosen as an effective dispersion medium for GNPs compared to other solvents [25,26]. The first dispersion treatment was a $1 \mathrm{~h}$ agitation in an ultrasonic bath. The second and third agitation treatments were performed using a higher power ( $750 \mathrm{~W}$ maximum power) ultrasound probe KT-600 (Kontes, Inc., Vineland, NJ) at $20 \mathrm{kHz}$ and $95 \%$ amplitude, for two different total durations of 20 and $40 \mathrm{~min}$, in time intervals of $5 \mathrm{~min}$ to avoid heating of the suspension to over $30^{\circ} \mathrm{C}$.

\subsubsection{GNP/3YTZP ceramic composite}

To evaluate the effect of GNPs in the ceramic matrix, monolithic 3YTZP was prepared from ceramic 3YTZP powders sized at $40 \mathrm{~nm}$ supplied by Tosoh Corporation (Tokyo, Japan). The as-received powders and powders annealed at $850^{\circ} \mathrm{C}$ for $30 \mathrm{~min}$ in air [27] were used to sinter the monolithic 3YTZP ceramics by SPS in a furnace model $515 \mathrm{~S}$, Dr. Sinter, Inc. (Kanagawa, Japan) at $1250{ }^{\circ} \mathrm{C}$ and 75 $\mathrm{MPa}$ for 5 and $7 \mathrm{~min}$, with 300 and $50^{\circ} \mathrm{C} / \mathrm{min}$ heating and cooling ramps, respectively. Graphite moulds of $1.5 \mathrm{~cm}$ inner diameter coated with graphite paper were used, in addition to graphite rods. Graphite tissue surrounded the moulding system to avoid heat losses. Approximately $3 \mathrm{~g}$ of powder were used for each pellet. Sintered cylindrical pellets of $15 \mathrm{~mm}$ diameter were manually sanded with P80 sandpaper to remove graphite from the SPS moulding system.

Composites with $1,2.5,5$ and 10 vol\% GNP content were prepared by adding the corresponding quantity of $3 \mathrm{YTZP}$ ceramic powder to the isopropanol GNP sonicated dispersion. The mixtures were further sonicated for $5 \mathrm{~min}$ with the probe and then dried on a hot plate with continuous magnetic stirring. The resulting powders were homogenized in an agatha mortar and sintered by SPS using the conditions described above. 


\subsection{Characterization of the starting powders and sintered specimens}

Elemental microanalysis (Elemental Analyzer TruSpec micro LECO) was performed to evaluate the composite powders prior to sintering to assess the total $\mathrm{C}$ content (and consequently, the measured GNP content) in the composites. Approximately 1-3 mg of the composite powders from each composition were placed in Sn capsules and completely burned in a pure oxygen environment at temperatures between $100-1000^{\circ} \mathrm{C}$. The combustion product $(\mathrm{CO} 2)$ was quantified by an infrared cell. This study was performed at Microanalysis General Service; Centros de Investigación, Tecnología e Innovación de la Universidad de Sevilla (CITIUS).

The density of the monolithic 3YTZP ceramics and composites was measured with the Archimedes method using distilled water as the immersion medium. The theoretical density of the composites was calculated using the rule of mixtures, considering $\rho=6.05 \mathrm{~g} \mathrm{~cm}-3$ for 3 YTZP and $\rho=2.2 \mathrm{~g} \mathrm{~cm}-3$ for GNPs (data from the suppliers), using measured GNP content volume fractions.

\subsubsection{Microstructural characterization}

X-ray diffraction tests were performed on the 3YTZP as-received and annealed powders, as well as on the sintered pellets (monolithic 3YTZP ceramics and composites with GNPs) to evaluate the different phases of zirconia. Quantitative estimation of the zirconia crystallographic phase content was obtained with the Rietveld refinement [28] using the TOPAS software [29]. The X-ray diffractometer was a D8 Advance A25 (Bruker Co. Massachusetts, USA) from CITIUS, with $\Theta: \Theta$ configuration, using a Bragg-Brentano geometry with a $0.5^{\circ}$ fixed divergence slot and a $\mathrm{Ni}$ filter to remove $\mathrm{k} \_\beta$ radiation from the $\mathrm{X}$-ray tube with the $\mathrm{Cu}$ anode. The selected parameters were a $2 \Theta$ scanning range from $10^{\circ}$ to $120^{\circ}$, a $0.015^{\circ}$ angular increment and a $0.1 \mathrm{~s}$ acquisition time. The tube was set at $40 \mathrm{kV}$ and 30 $\mathrm{mA}$, and the specimen was rotated at 30 r.p.m.

Raman spectroscopy and high resolution scanning electron microscopy (HRSEM) were used to assess the most suitable deagglomeration method for the asreceived GNPs in isopropanol. For this purpose, a few droplets of the sonicated suspensions were deposited over a glass slide for Raman spectroscopy or on a grid for HRSEM (Hitachi S5200, CITIUS) inspection after drying.

The presence and structural integrity of the GNPs in the sintered composites after the dispersion treatments, as well as the existence of other carbon allotropes, were assessed by Raman vibrational spectroscopy on the fractured surfaces of the composites. Five to eight spectra from each specimen were taken and using a Raman Horiba Jobin Yvon LabRaman HR800 with Olympus BX 41optic system and the Labsec 5.25.15 data acquisition software (Instituto de Ciencia de Materiales de Sevilla, ICMSE). The Raman spectrometer used a green HeNe laser $(532.1 \mathrm{~nm})$ at $20 \mathrm{~mW}$ power as the source, with a diffraction grid of 600 lines per $\mathrm{mm}$ and a 100x objective with a $100 \mu \mathrm{m}$ confocal aperture.

The distribution assessment of the GNPs in the 3YTZP matrix was carried out by scanning electron microscopy (SEM, JEOL 6460LV, CITIUS) of the composites polished with up to a $1 \mu \mathrm{m}$ diamond paste. The GNP distribution was assessed on two sections of each composite, both parallel and perpendicular to the SPS compression axis, to account for any degree of anisotropy. The ceramic grain size was estimated as the equivalent planar diameter, $d=2(\operatorname{area} / \pi) 1 / 2$ by HRSEM on the polished samples annealed in air at $1150^{\circ} \mathrm{C}$ for $15 \mathrm{~min}$ to reveal the grain boundaries. Approximately 300 grains were measured for each specimen. The shape factor of the ceramic grains was estimated as $F=4 \pi$ area/(perimeter)2. The ImageJ and Origin software were used to quantify these parameters.

\subsubsection{Hardness and electrical conductivity}

The hardness of the composites and monolithic 3 YTZP ceramic was evaluated with a Vickers Struers Duramin micro-indenter to assess the influence of the GNP content on the room temperature mechanical properties. Indentation tests were carried out on the polished top surfaces and cross sections to account for the structural anisotropy due to the GNP geometry and to determine the effect of the uniaxial stress applied during the sintering on the GNP alignment. At least 20 microindentations were analysed, sufficiently separated from each other to avoid overlapping the stress fields and to avoid border effects, with loads of $1.96 \mathrm{~N}$ applied for $15 \mathrm{~s}$. 

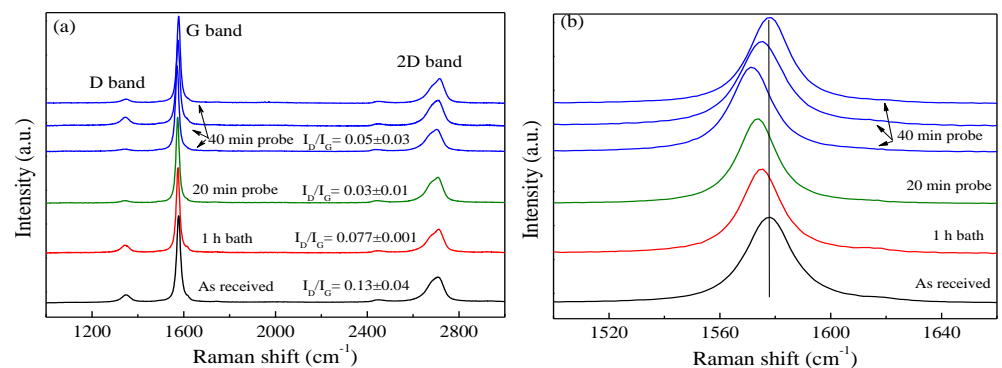

The two-point DC conductivity of GNP/3YTZP composites was estimated with a model 2000 Keithley multimeter and an in-house measuring cell in paralellepipedic specimens. Electrodes were made by coating selected specimen faces with a colloidal

Fig. 1. (a) Raman spectra of the GNPs subjected to different ultrasonication . 1. (a) Raman spectra of the GNPs subjected to different ultras silver varnish and annealed up to $650^{\circ} \mathrm{C}$ for $30 \mathrm{~min}$ with a $3{ }^{\circ} \mathrm{C} / \mathrm{min}$ heating ramp in Ar to avoid GNP degradation. Conductivity was measured in two directions (parallel and perpendicular to the SPS compression axis) to account for the GNP preferential orientation.

\section{Results and discussion}

\subsection{GNP de-agglomeration}

To have well dispersed and undamaged GNPs in the composites, the microstructure of the GNPs after sonication in isopropanol for different time intervals and with different energies was first analysed. Fig. 1 presents the Raman spectra for the three dispersion routines detailed in the experimental procedure. The GNP characteristic D, G and 2D bands are located at $\sim 1350, \sim 1575$ and $\sim 2700 \mathrm{~cm}^{-1}$, respectively. These values are similar to those given in the literature for graphitic materials [30] and also for GNP/ceramic composites with a silicon nitride matrix [25]. These bands confirm the structural integrity of GNPs after the dispersion treatments. The 2D band, which gives information relative to the pile-up ordering of the graphene sheets, shows a lower intensity than the $G$ band and can be de-convoluted into several peaks, which confirms that our GNPs are composed of several graphene sheets $[31]$. The $I_{D} / I_{G}$ ratio is often used as an indicator of the defect population in graphitic materials, since the $\mathrm{D}$ band is associated with the crystal disorder or defects in these materials (disordered-induced band) $[32,33]$. The ID/IG ratio is also inversely proportional to the inplane crystallite size [30]. The Calculated average of the ID/IG for the as-received GNPs is $0.13 \pm 0.04$, while for the GNPs subjected to a $1 \mathrm{~h}$ ultrasonic bath, 20 and $40 \mathrm{~min}$ with the ultrasonic probe, the ID/IG values are $0.077 \pm 0.001,0.03 \pm 0.01$ and $0.05 \pm 0.03$, respectively. The fact that the $\mathrm{I}_{\mathrm{D}} / \mathrm{I}_{\mathrm{G}}$ values do not differ much from each other indicates that the crystalline structure was not damaged by the ultrasonic treatments. However, different Raman positions for the $G$ and 2D bands were detected for several spectra acquired from the GNPs subjected to 40 min of the ultrasound probe. These shifts are shown in fig. 1b, where three detailed Raman spectra for these GNPs can be observed with their $G$ maxima in different positions. This is in contrast to the data from the other two types of dispersed GNPs with reproducible Raman spectra and maxima fixed at $1575 \mathrm{~cm}^{-1}$ as described in the literature [25]. The G
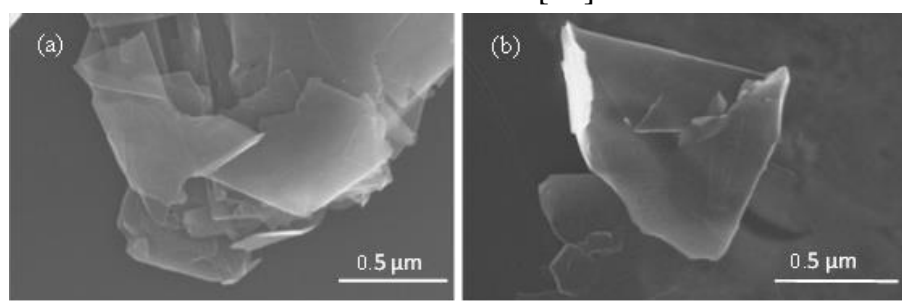

and 2D shifts (not shown) could be a sign of inhomogeneity, probably due to some GNP structural damage induced by the long sonication time. Therefore, the 40 min ultrasound probe treatment was

Fig. 2. HRSEM micrographs of the GNPs dispersed in isopropanol subjected to (a)

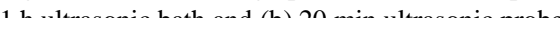

discarded for the GNPs dispersion. The slight decrease in the ID/IG for the $20 \mathrm{~min}$ probe may indicate the improved GNP dispersion (with a smaller crystallite size than aggregated GNPs).

The HRSEM micrographs of the dispersed GNPs (fig. 2a) show some agglomerates for the GNPs sonicated for $1 \mathrm{~h}$ at a low energy (ultrasound bath), 
while the GNPs treated with the ultrasound probe for 20 min (fig 2b) have an improved dispersion, leaving only small GNP pile-ups. This result points to this final sonication treatment as the ideal candidate to obtain a better homogeneity in the ceramic composites. It can be observed that the GNPs are rather flat, mostly with smooth straight edges and no crumpling, with a very small degree of edge folding and bending.

\subsection{Processing conditions for the 3 YTZP ceramic matrix}

The processing approach for monolithic 3YTZP is geared at testing the effects of two aspects from the sintered bodies' density and phases: a) the use of as-received commercial ceramic powder versus the annealed powder and b) the holding time conditions during SPS, 5 verse $7 \mathrm{~min}$. The goal is to obtain a fully tetragonal zirconia matrix and fully dense sintered specimens.

Absolute and relative densities of the sintered monolithic ceramics are indicated in table 1. All the ceramics in the table present high relative density. However, the 3YTZP ceramics sintered from annealed powders are denser than the specimens prepared from the as-received powders, and exhibit nearly the same theoretical density $(100 \%$ versus $98.7 \%$ for 5 min sintering time and $99.6 \%$ versus $98.0 \%$ for $7 \mathrm{~min}$ sintering time, respectively). Sintering for longer periods ( $7 \mathrm{~min}$ versus $5 \mathrm{~min}$ ) does not increase the density, but instead gives a slightly lower density, which is within experimental uncertainty.

Crystallographic phases for the 3YTZP ceramics sintered for different holding times and their corresponding starting powders can be compared by their XRD patterns, as shown in figure 3.

The main phase in each analysed specimen is the reduced tetragonal phase (ZrO1.95) (JCPDS 01-0811544). The as-received powder presents pronounced monoclinic peaks (fig. 3a) at $2 \Theta=28.2^{\circ}$ and $31.5^{\circ}$ (JCPDS 01-078-1807), which disappear in the annealed powders (fig. 3b) and in the sintered ceramics. In each pattern, the main tetragonal peak $\left(2 \Theta=30.2^{\circ}\right)$ is non-symmetric and more broad on the left side. This is due to a contribution of the cubic zirconia phase (JCPDS 01-082-1246) in the powders and to the contribution of a solid solution $\mathrm{Zr}_{1-\mathrm{x}} \mathrm{Y}_{\mathrm{x}} \mathrm{O}_{2-}$ $x / 2$ in the sintered ceramics. The composition and structure of this phase has been determined by Rietveld refinement in each case, and corresponds to cubic $\mathrm{Zr}_{0.80} \mathrm{Y}_{0.20} \mathrm{O}_{1.9}$ (JCSD 01-082-1246) for all the sintered specimens. No significant differences can be
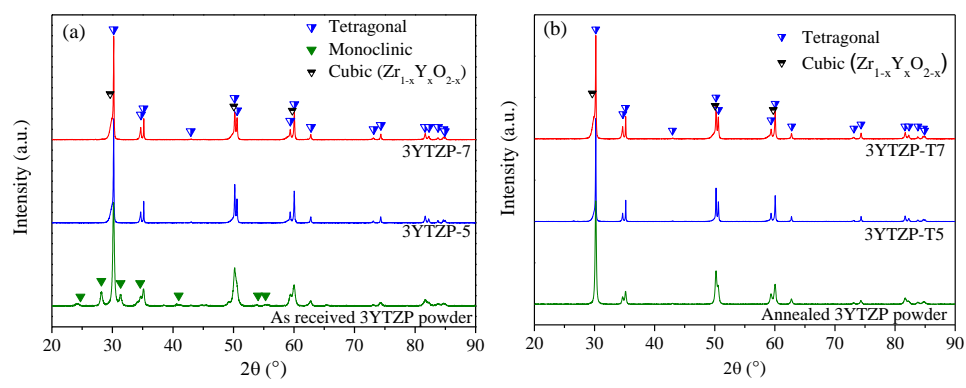

detected between the XRD patterns of the four sintered 3 YTZP monoliths, as shown in figure 3 (a) and (b).

Fine microstructures with grain sizes $d<300 \mathrm{~nm}$ were observed for all sintering conditions (see table $1)$, due to the short holding times used in the SPS.

Fig. 3. XRD patterns of the 3 YTZP ceramic powders (a) as-received and (b) The grain size indicated in table 1 was estimated from the histograms fitted to a log-normal distribution for each sintered 3YTZP ceramic. The histograms for the ceramics sintered for longer times (7 min) were skewed more to the left, and showed better fits, with higher squared correlation factors (0.96 and 0.95 versus 0.91 and 0.89 , respectively), indicating a more stable grain size distribution. Some pores were detected by HRSEM observations in the 3YTZP ceramics sintered from the as-received powders, especially in the specimen sintered for longer times $(7 \mathrm{~min})$, in agreement with the density results (table 1). The grains are equiaxed, with a rounded shape in all specimens and with shape factor $\mathrm{F}=0.7 \pm 0.1$.

To summarize, using the annealed starting powder and an SPS holding time of $5 \mathrm{~min}$ are considered as the most adequate processing conditions to extrapolate composites with the GNP. This determination is based on the superior density results and the absence of the monoclinic phase in the 
monolithic 3YTZP-T5 (see table 1) sample. The microstructure did not improve for longer sintering times.

\subsection{GNP/3YTZP composite microstructure.}

The elemental microanalysis results, shown in table 2 , indicate that the measured GNP content for the composites is very similar to the nominal

Table 1 Processing conditions, density and grain size of monolithic 3YTZP ceramics

\begin{tabular}{|c|c|c|c|c|c|c|}
\hline Sample & $\begin{array}{l}\text { Powder } \\
\text { treatment }\end{array}$ & $\begin{array}{l}\text { SPS Holding time } \\
(\min )\end{array}$ & $\begin{array}{l}\square\left({ }^{g} \mathrm{~cm}^{3}\right) \\
\pm 0.1\end{array}$ & $\begin{array}{l}\square \square \text { relative (\%) } \\
\pm 2\end{array}$ & $\mathrm{~d}(\mu \mathrm{m})$ & s.d. $(\mu \mathrm{m})$ \\
\hline 3YTZP-5 & - & 5 & 6.0 & 99 & 0.26 & 0.02 \\
\hline 3YTZP-7 & - & 7 & 6.0 & 98 & 0.23 & 0.01 \\
\hline 3YTZP-T5 & Annealed & 5 & 6.1 & 100 & 0.29 & 0.02 \\
\hline 3YTZP-T7 & Annealed & 7 & 6.0 & 100 & 0.22 & 0.01 \\
\hline
\end{tabular}

Table 2 Real GNP content, density and grain size parameters for GNP/ceramic composites and monolithic 3YTZP sintered in identical conditions

\begin{tabular}{|c|c|c|c|c|c|}
\hline Sample & Real GNP (vol\%) \pm 0.1 & $\square\left({ }^{8} \mathrm{Cm}^{2}\right) \pm 0.1$ & $\square$ relative $(\%) \pm 2$ & $\mathrm{~d}(\mu \mathrm{m})$ & s.d. $(\mu \mathrm{m})$ \\
\hline 3YTZP-T5 & 0 & 6.1 & 100 & 0.29 & 0.02 \\
\hline C1-GNP & -- & 6.0 & 100 & 0.27 & 0.13 \\
\hline C2.5-GNP & 2.2 & 6.0 & 100 & 0.22 & 0.10 \\
\hline C5-GNP & 4.4 & 5.8 & 99 & 0.21 & 0.09 \\
\hline C10-GNP & 9.5 & 5.7 & 100 & 0.25 & 0.11 \\
\hline
\end{tabular}

GNP content. There were only small $(0.3-0.5$ vol\%) GNP losses during the processing of the $2.5,5$ and 10 vol\% GNP composites. However, the elemental microanalysis of the composite with a nominal 1 vol\% GNP did not give a reliable result, possibly because the $\mathrm{C}$ content for this composite was close to the equipment detection threshold. The density of the composites is close to $100 \%$ of the theoretical predictions, as indicated in table 2. These values are higher values than those reported by Chen et al. for GNP/3YTZP composites with lower a GNP content but SPSed at similar temperatures [23], who found a decrease in the density with the increasing GNP content.

Although the main phase for the composites, the monolithic 3YTZP-T5, and the starting ceramic powders is the reduced tetragonal zirconia, the XRD patterns (figure 4) reveal the presence of the monoclinic phase in the as-sintered composites with a GNP content higher than 2.2 vol\%. Other authors have also observed an increase of the monoclinic phase in $\mathrm{ZrO}_{2}$ with the addition of graphene [22]. The main graphite peak (JCPDS 00-026-1076, 20=26.6 $)$ was also detected in composites with 5 and $10 \mathrm{vol} \%$ nominal GNP, with the $10 \%$ sample peak more clearly visible in the XRD patterns (fig. 4).

Raman spectra from the $20 \mathrm{~min}$ probe sonicated GNP samples and the sintered GNP/3YTZP composites, represented in figure 5 , show $\mathrm{D}, \mathrm{G}$ and 2D characteristic bands for the GNPs, located at $\sim 1350, \quad \sim 1580-1585$ and $\sim 2710-2715 \quad \mathrm{~cm}^{-1}$, respectively. This confirms the GNP survival after the dispersion treatment and the SPS consolidation. The $\mathrm{G}$ and $2 \mathrm{D}$ bands in the spectra acquired from the composites are displaced towards

Fig. 4. XRD patterns of the GNP/3YTZP composites with 1

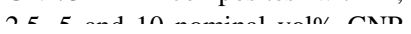
higher frequencies with respect to the GNP spectrum,

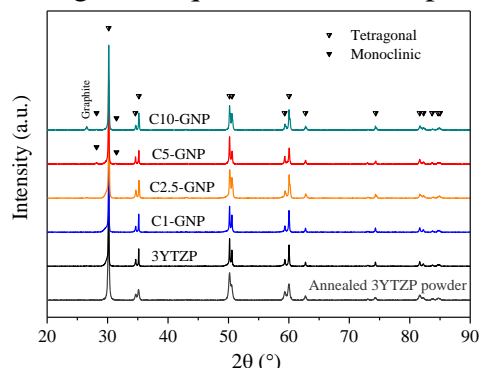
which has been attributed by previous authors to 
the residual stresses imposed to the GNPs by the ceramic matrix [34].

The small increase in the $\mathrm{I}_{\mathrm{D}} / \mathrm{I}_{\mathrm{G}}$ ratio observed in the composites with respect to the GNP $(0.03 \pm 0.01$ to $0.17 \pm 0.13$ ), see figure 5 , points to a negligible damage of the GNPs during sintering. However, this ratio decreases monotonically for the increasing GNP content (from $0.17 \pm 0.13$ for the $\mathrm{C} 1-\mathrm{GNP}$ to $0.10 \pm 0.05$ for the C10-GNP composite). This decrease is unexpected and has not been reported previously. Instead, we expect a similar disorder

crumpling of the GNP borders and are therefore detected as more perfect structures. In fact, it has been shown that the GNP borders are detected as defects by Raman spectroscopy, therefore increasing the D band peak $[35,36]$.

The GNP distribution in the ceramic matrix has been analyzed for the composites in two relevant surfaces: perpendicular (top surface or in-plane) and parallel (cross section) to the sintering compression

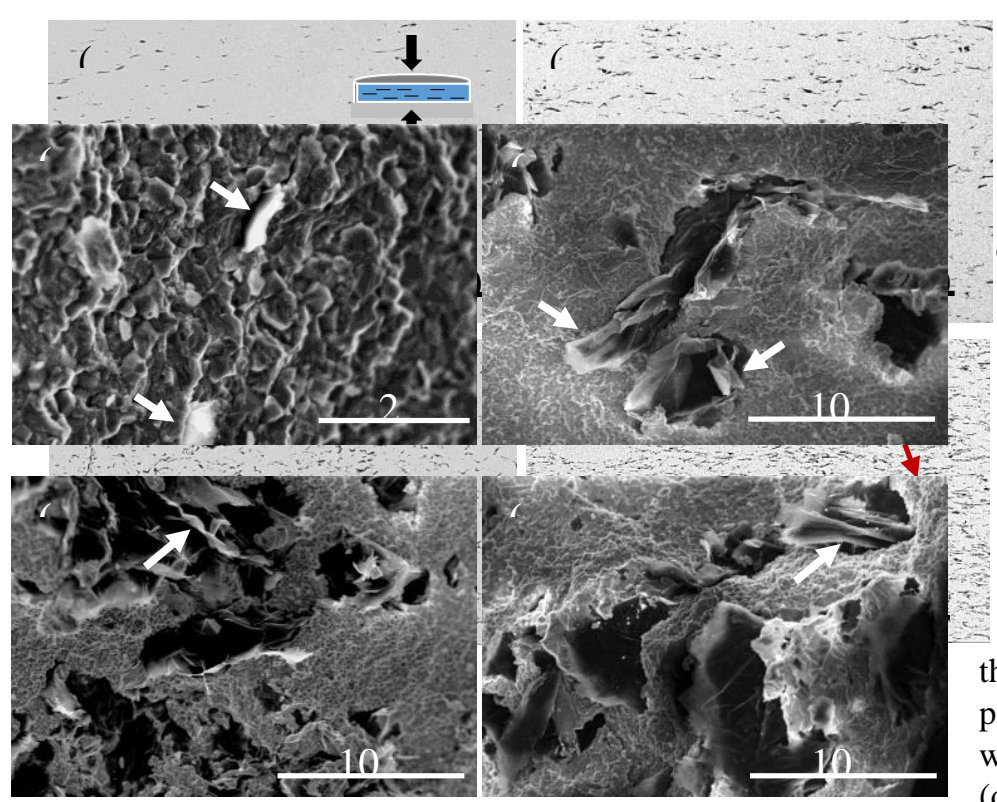

degree in composites with an increasing GNP content, while this result indicates less disordered graphene in composites with a higher GNP content. This could be attributed to the inplane interconnection of the GNPs, which forms graphene structures with higher areas (higher in plane crystallite size). This can minimize the bending or axis. Low magnification, scanning electron micrographs using backscattered electrons were used to image the previously mentioned polished surfaces. The micrographs show a light phase corresponding to the 3YTZP matrix and a dark phase corresponding to the GNPs (figure 6). A nearly homogeneous GNP distribution throughout the ceramic matrix is observed, with scarce and small $(<20 \mu \mathrm{m})$ GNP agglomerates or groups of interconnected GNPs, which grow larger for composites with a higher GNP content (figure 6).

stress applied during sintering and to the high aspect Raman spectra for the ratio and GNP/3YTZP composites stiffness of the GNPs. This result has been reported in the literature for GNP composites with different ceramic matrices [26,37-39]. Percolated networks of GNPs appear with increasing graphene content.

The composite fracture surfaces observed in the SEM show the GNPs characteristic shape and distribution in the ceramic matrix (figure 7). The GNP planar dimensions and width are aligned with the specified data from the supplier $\left(\mathrm{d}_{\text {planar }} \sim 5 \mu \mathrm{m}\right)$ and are significantly larger

The GNPs are aligned in a preferential orientation, with their major surface perpendicular to the SPS compression axis, exhibiting aligned segments along the cross sections (figure 6), and are randomly oriented in the top surface. This indicates a structural

Fig. 6 Backscattered scanning electron (BSE) micrographs of the composites cross anisotropy in the composites due to the uniaxial than the ceramic grains $(\mathrm{d}<0.3 \mu \mathrm{m})$. For this reason, the ceramic grains cannot be observed at the same scale used for GNP inspection. The GNPs are well dispersed in the 3YTZP matrix, both isolated (arrowed in fig. 7a) and forming small pile ups and larger overlapping interconnected groups ( $\mathrm{d} \sim 10 \mu \mathrm{m}$, arrowed in fig. 7d). Abundant GNP pull outs can

Fig. 7 Low magnification SEM micrographs of the fracture surfaces of the GNP/

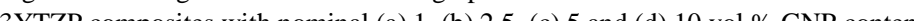


also be observed in the figure. Although some GNPs are flat (fig $7 \mathrm{a}$ and $\mathrm{d}$ ), others show crumpling (arrowed in fig 7b), bending (arrowed in fig 7b) and wavy surfaces (arrowed in fig 7c). However, no micro-fractures have been detected in the ceramic matrix. Low magnification scanning electron microscopy micrographs (figure 6) show a higher degree of interconnection between the GNPs in composites with increasing GNP content (in agreement with the Raman results) where some GNP aggregates can also be observed.
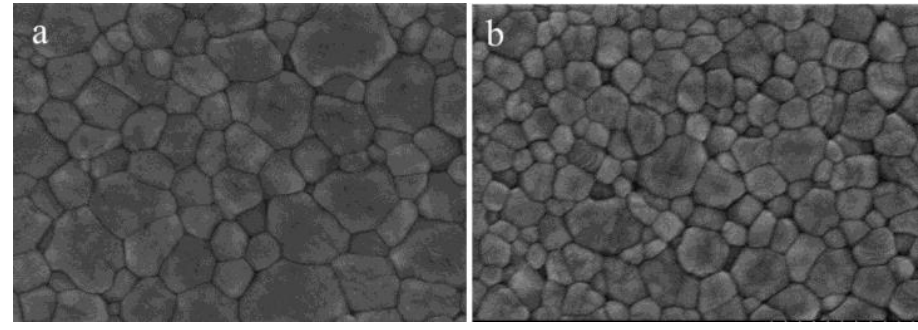

agglomeration

The ceramic grains are equiaxed in all the composites, with a shape factor $\mathrm{F}=0.72 \pm 0.07$, which is independent of the GNP content. However, a refinement effect of the ceramic grains due to GNPs has been detected (table 2). A remarkable decrease in the grain size (approximately 20\%) is observed when the GNP content increases from $\sim 1$ to $4.4 \mathrm{vol} \%$ in the composites $(0.27$ to $0.21 \mu \mathrm{m})$, as seen in figure 8 . This microstructure refinement due to the GNPs is similar to that obtained with other carbon nanostructures [40]. A homogenizing effect on the matrix grain size has also been detected, with narrower ceramic grain size distributions when the GNP content increases (standard deviation decreases from 0.13 to $0.09 \mu \mathrm{m}$ ). However, for the 9.5 vol\% GNP content, the grain size decrease is very small with respect to the monolithic 3YTZP ceramic. This may be since the

more important for a higher GNP content by reducing the GNP refinement effect on the ceramic matrix grain size. The grain size distribution for this

Fig. 8 HRSEM micrographs of the polished and revealed surfaces of the 3YTZP SPSed GNP/3YTZP composites with a similar GNP content by Chen et al. [23]. Other authors have also observed deterioration in the hardness for graphene/alumina composites with increasing composite is also wider than for the C5-GNP, indicating less homogeneity in the grain size, which can also be attributed to the GNP agglomeration. The increased processing efforts should be addressed to obtain a better microstructural homogeneity in high content GNP/3YTZP composites.

\subsection{Hardness and electrical conductivity of GNP/3YTZP composites}

The Vickers hardness of the sintered GNP/3YTZP composites is indicated in table 3. The average Vickers hardness of the composites with a low GNP content (nominal $1 \mathrm{vol} \%$ ) is slightly higher than that corresponding to the monolithic ceramic 3YTZP (3\% increase). However, the Vickers hardness decreases to a lower level (by $16 \%$ and $42 \%$ with respect to the monolithic value) for higher GNP content (higher than 2.2 vol\% GNP). The values for a small graphene graphene content above a certain threshold value $[41,42]$. For the SPSed GNP/ZrO2 composite, the decrease in the micro-hardness for GNP concentrations $\geq 0.05 \mathrm{wt} \%$ was attributed to the induced grain yielding and slipping [43]. In our study, the decrease in the hardness is thought to be caused by the increased contribution of the GNP softer phase in the composites, since it has been reported that the hardness for the SPSed multilayered bulk graphene, obtained using the nanoindentation method, is less than $1 \mathrm{GPa}$ [44], ten times smaller than for the 3YTZP. The presence of monoclinic zirconia detected for a GNP content higher than 2.2 vol\% can also decrease the hardness of the composites. The slight increase in hardness for a low GNP content is most likely linked to the grain size refinement. 
Table 3 Vickers hardness and electrical conductivity of GNP/3YTZP composites and monolithic 3YTZP for comparison

\begin{tabular}{cccccc}
\hline Sample & $\begin{array}{c}\mathrm{H}_{\text {in plane }} \\
(\mathrm{GPa})\end{array}$ & $\begin{array}{c}\mathrm{H}_{\text {cross section }} \\
(\mathrm{GPa})\end{array}$ & $\begin{array}{c}\mathrm{H}_{\text {average }} \\
(\mathrm{GPa})\end{array}$ & $\sigma_{\|}\left(\mathrm{S} \cdot \mathrm{m}^{-1}\right)$ & $\sigma_{\perp}\left(\mathrm{S} \cdot \mathrm{m}^{-1}\right)$ \\
\hline 3YTZP-T5 & -- & $13.9 \pm 0.5$ & $13.9 \pm 0.5$ & -- & -- \\
C1-GNP & $14.2 \pm 0.6$ & $14.5 \pm 0.3$ & $14.4 \pm 0.6$ & -- & -- \\
C2.5-GNP & $13.0 \pm 0.7$ & $13.3 \pm 0.5$ & $13.2 \pm 0.7$ & $13.5 \pm 0.8$ & $38.3 \pm 0.6$ \\
C5-GNP & $11.3 \pm 0.9$ & $12.0 \pm 1.1$ & $11.7 \pm 1.1$ & $16.4 \pm 0.1$ & $111.5 \pm 1.1$ \\
C10-GNP & $7.7 \pm 1.4$ & $8.5 \pm 0.8$ & $8.1 \pm 1.4$ & & - \\
\hline
\end{tabular}

The difference between the in plane and cross section hardness values is linked to the microstructural anisotropy produced by the GNP geometry and their preferential orientation, due to the uniaxial pressure applied during sintering. This difference logically increases when the GNP content increases. A similar fact occurred in the carbon nanotube/ceramic composites sintered by SPS when the carbon nanotube agglomerates were aligned in preferential orientations due to uniaxial pressure during sintering $[45,46]$. The lower hardness values occur on the top surfaces when the indenter falls on the larger GNP in-plane surface. This suggests a low energy microscopic deformation mechanism, as sliding, which has been identified as the main microscopic mechanism for indentation on top surfaces for bulk graphene nanoplatelets [47]. Other mechanisms, such as wrinkling, kinking and GNP pull-out (which can be observed in fig. 7), are also characteristic of indentation on top surfaces. The higher hardness values are obtained when the indenter lies on cross sections, where GNPs are out of plane (fig. 6). This can be due to the intrinsic properties of the GNPs, such as the increased bending rigidity in the principal (in-plane) direction [15], although the extremely high Young's modulus reported for monolayer graphene decreases with the increasing number of layers [48]. Our results are also in agreement with authors [49] who have reported that the indentation load required to achieve a certain displacement is much higher when the indentation occurs on the orthogonal surface of an SPSed multilayered graphene surface than when it occurs on the top surfaces. This is attributed to the microscopic mechanisms operating in the cross sections, different than the those corresponding to indentation on the top surfaces, such as bending, push-out and pop-out, which produce separation and cracking. The opposite behaviour has been observed in porous bulk graphene. Since the hardness is a measure of the resistance to deformation, a porous transverse surface presents a lower hardness than the top surface $(0.61 \pm 0.13 \mathrm{GPa}$ on the top surface versus $0.28 \pm 0.02$ GPa on the transverse surface) [44].

Regarding electrical conductivity, it is well-known that the 3YTZP is electrically isolating at room temperature. The addition of low GNP percentages, up to a 2.2 vol\% GNP does not allow electrical conductivity, but a 4.4 vol\% GNP converts the composite to be electrically conductive (table 3 ). This implies the electrical percolation threshold is situated between these two percentages of 2.2 and $4.4 \mathrm{vol} \%$ GNP. This limit is similar to the published values for rGO/Si3N4 (< 4.3 vol\%) [50], rGO/3YTZP (2.5 vol\%) [18] and for graphene nanosheets $/ \mathrm{Al}_{2} \mathrm{O}_{3}$ (3 vol\%) [51] and lower than the published values for $\mathrm{GNP} / \mathrm{Si}_{3} \mathrm{~N}_{4}$ composites, where the electrical percolation occurs between 7 and 9 vol\% [38]. The electrical conductivity obtained in this work for a 9.5 vol\% GNP/3YTZP, $\sim 111 \mathrm{~S} \mathrm{~m}^{-1}$ is also much higher than the values published for Si3N4 with an 11 vol\% GNP $\left(12 \mathrm{~S} \mathrm{~m}^{-1}\right)$ [38], although it is lower than the values obtained for PLS sintered GNP/3YTZP, $280 \mathrm{~S}$ $\mathrm{m}^{-1}$ for $2 \mathrm{wt} \%$ GNP [24]. Factors, such as the presence of functional groups on graphene sheets, aspect ratio of the graphene sheets, inter-sheet junctions, distribution in the matrix, wrinkles and folds, processing methods, etc., influence the electrical conductivity and the percolation threshold of the composites [8]. The rGO's typical dimensions of several hundred nm [18], although wrinkled, are the reason for a better distribution into the ceramic matrix and for the increased electrical conductivity of 
the rGO/3YTZP composites, while the dimensions of the GNPs used in this study are much larger than the ceramic grains. However, the GNP/3YTZP composites processed in this work still have the potential for applications where electrical conductivity is required. The electrical conductivity presents significant anisotropy, as previously shown by other authors $[36,50,52]$. This anisotropy increases with the GNP content with a $3 x$ ratio between the conductivities in the parallel and perpendicular directions with respect to the SPS compression axis for the $4.4 \mathrm{vol} \%$ GNP and a $7 \mathrm{x}$ ratio for the $9.5 \mathrm{vol} \%$. The higher conductivity occurs in the direction perpendicular to SPS compression axis, where the aligned GNPs present their major surface. This electrical anisotropy is a consequence of two effects. First, the graphene exhibits intrinsic electrical anisotropy. It has a significantly higher conductivity in the a-b plane than along the $\mathrm{c}$ axis [53], resulting in a higher current density when measuring in an inplane geometry, where current flows preferentially on the graphene planes forming the GNPs. Second, the preferential orientation of the GNPs due to the uniaxial compression applied during the sintering facilitates the conductivity in this direction. When above the percolation threshold, the electrical conductivity increases with the GNP content in the two measured orientations. The increase is much higher in the perpendicular direction to the compression axis during sintering (more than double), while the increase is only slight in the parallel direction. This increase is similar to that found for GNP composites with a silicon nitride ceramic matrix [37], where a fourfold increase in the effective current was found in a composite when the GNP content increased from 12 to 15 wt. $\%$. These authors also reported a conductivity value of $\sim 4100 \mathrm{~S}$ $\mathrm{m}^{-1}$ for a 24 vol\% GNP content [50]. Future works will be carried out to explore the conductivity in the GNP/3YTZP composites with a higher GNP content and optimized dispersion.

\section{Conclusions}

In this work, fully dense yttria tetragonal zirconia monolithic ceramics and highly anisotropic conductive ceramic composites with cost-effective graphene nanoplatelets have been fabricated by wet powder processing and spark plasma sintering.

Ultrasonic probe agitation of the graphene nanoplatelets in isopropanol for $20 \mathrm{~min}$ has proven effective in separating the GNPs, leaving only small pile ups or aggregates and avoiding their degradation. The combination of annealing the 3YTZP commercial powders prior to densification and sintering at $1250^{\circ} \mathrm{C}$ for 5 min produced fully dense composites with submicrometric equiaxed ceramic grains and much larger micrometric GNPs dispersed in the ceramic matrix. The ultrasonic dispersion treatment was successful for low GNP content, as only a low presence of small sized GNP agglomerates was observed in the composites when increasing the GNP content. The GNPs were oriented preferentially in the plane perpendicular to the compression axis during sintering (in-plane orientation). A grain size refinement effect was observed due to the GNPs, with a decrease in the grain size as the GNP content increased.

The Vickers hardness increased slightly for low GNP content, but decreased in composites with an increasing GNP content (2.2 - 9.5 vol\%), most likely due to the increased presence of the softer GNP phase and to the monoclinic zirconia content detected for composites with more than a 2.2 vol\% GNP content. The composite hardness showed a difference between the "in plane" (weaker) and "cross section" (harder) values. This difference grows when the GNP content increases and is related to the microstructural anisotropy due to the preferential GNP orientation and the special geometry combined with the easy delamination (sliding) of the GNPs under shear stresses when the indenter presses into the top surface.

The electrical percolation threshold for the composites is between 2.2 and 4.4 vol\% GNP content. Additionally, the electrical conductivity increases with the GNP content, due to their higher interconnection and to the improved crystallinity of the GNPs measured by the $I_{D} / I_{G}$ ratio from the Raman spectra. The effect of anisotropy on the electrical conductivity is significant through the combination of two effects: the higher "in plane" 
GNP conductivity and the higher "in plane" GNP interconnection due to their preferential orientation. This anisotropy increases with the increasing GNP content.

\section{Acknowledgments}

The authors acknowledge the financial support provided by the Spanish Ministry, Ministerio de Economía y Competitividad, under project MAT2015-67889-P, co-funded by European FEDER.

\section{References}

[1] R.C. GARVIE, R.H. HANNINK, R.T. PASCOE, Ceramic steel?, Nature. 258 (1975) 703-704. doi:10.1038/258703a0.

[2] C. Piconi, G. Maccauro, Zirconia as a ceramic biomaterial, Biomaterials. 20 (1999) 1-25. doi:10.1016/S01429612(98)00010-6.

[3] V. Dhand, K.Y. Rhee, H.J. Kim, D.H. Jung, A comprehensive review of graphene nanocomposites: research status and trends, J. Nanomater. 2013 (2013) 158

[4] A.C. Ferrari, F. Bonaccorso, V. Fal'ko, K.S. Novoselov, S. Roche, P. Bøggild, S. Borini, F.H.L. Koppens, V. Palermo, N. Pugno, J.A. Garrido, R. Sordan, A. Bianco, L. Ballerini, M. Prato, E. Lidorikis, J. Kivioja, C. Marinelli, T. Ryhänen, A. Morpurgo, J.N. Coleman, V. Nicolosi, L. Colombo, A. Fert, M. Garcia-Hernandez, A. Bachtold, G.F. Schneider, F. Guinea, C. Dekker, M. Barbone, Z. Sun, C. Galiotis, A.N. Grigorenko, G. Konstantatos, A. Kis, M. Katsnelson, L. Vandersypen, A. Loiseau, V. Morandi, D. Neumaier, E. Treossi, V. Pellegrini, M. Polini, A. Tredicucci, G.M. Williams, B. Hee Hong, J.-H. Ahn, J. Min Kim, H. Zirath, B.J. van Wees, H. van der Zant, L. Occhipinti, A. Di Matteo, I.A. Kinloch, T. Seyller, E. Quesnel, X. Feng, K. Teo, N. Rupesinghe, P. Hakonen, S.R.T. Neil, Q. Tannock, T. Löfwander, J. Kinaret, Science and technology roadmap for graphene, related two-dimensional crystals, and hybrid systems., Nanoscale. 7 (2015) 4598-4810. doi:10.1039/c4nr01600a.

[5] S. Bai, X. Shen, Graphene-inorganic nanocomposites, Rsc Adv. 2 (2012) 64-98. doi:10.1039/c1ra00260k

[6] H. Fukushima, L. Drzal, Graphite nanoplatelets as reinforcements for polymers: structural and electrical properties, 17th Annu. Conf. .... (2002).

[7] B.T. (Brian T. Kelly, Physics of graphite, Applied Science, 1981.

[8] V. Singh, D. Joung, L. Zhai, S. Das, S.I. Khondaker, S. Seal, Graphene based materials: Past, present and future, Prog. Mater. Sci. 56 (2011) 1178-1271. doi:10.1016/j.pmatsci.2011.03.003.
[9] J.N. Coleman, Liquid-Phase Exfoliation of Nanotubes and Graphene, Adv. Funct. Mater. 19 (2009) 3680-3695. doi:10.1002/adfm.200901640.

[10] S. Bae, H. Kim, Y. Lee, X. Xu, J.-S. Park, Y. Zheng, J Balakrishnan, T. Lei, H. Ri Kim, Y. Il Song, Y.-J. Kim, K.S. Kim, B. Ozyilmaz, J.-H. Ahn, B.H. Hong, S. Iijima, Roll-toroll production of 30-inch graphene films for transparent electrodes, Nat Nano. 5 (2010) 574-578.

[11] J. Cai, P. Ruffieux, R. Jaafar, M. Bieri, T. Braun, S. Blankenburg, M. Muoth, A.P. Seitsonen, M. Saleh, X. Feng, Atomically precise bottom-up fabrication of graphene nanoribbons, Nature. 466 (2010) 470-473.

[12] V. Palermo, Not a molecule, not a polymer, not a substrate... the many faces of graphene as a chemical platform, Chem. Commun. 49 (2013) 2848-2857. doi:10.1039/C3CC37474B.

[13] A.S. Mayorov, R. V. Gorbachev, S. V. Morozov, L. Britnell, R. Jalil, L.A. Ponomarenko, P. Blake, K.S. Novoselov, K. Watanabe, T. Taniguchi, A.K. Geim, Micrometer-Scale Ballistic Transport in Encapsulated Graphene at Room Temperature, Nano Lett. 11 (2011) 2396-2399. doi:10.1021/nl200758b.

[14] A.A. Balandin, S. Ghosh, W. Bao, I. Calizo, D Teweldebrhan, F. Miao, C.N. Lau, Superior thermal conductivity of single-layer graphene., Nano Lett. 8 (2008) 902-7. doi:10.1021/n10731872.

[15] C. Lee, X. Wei, J.W. Kysar, J. Hone, Measurement of the Elastic Properties and Intrinsic Strength of Monolayer Graphene, Science (80-. ). 321 (2008)

[16] L.-A. Liew, V.M. Bright, R. Raj, A novel micro glow plug fabricated from polymer-derived ceramics: in situ measurement of high-temperature properties and application to ultrahigh-temperature ignition, Sensors Actuators A Phys. 104 (2003) 246-262. doi:10.1016/S0924-4247(03)00027-X.

[17] S. Stankovich, D.A. Dikin, R.D. Piner, K.A. Kohlhaas, A. Kleinhammes, Y. Jia, Y. Wu, S.T. Nguyen, R.S. Ruoff, Synthesis of graphene-based nanosheets via chemical reduction of exfoliated graphite oxide, Carbon N. Y. 45 (2007) 1558-1565. doi:10.1016/j.carbon.2007.02.034.

[18] J.-H. Shin, S.-H. Hong, Fabrication and properties of reduced graphene oxide reinforced yttria-stabilized zirconia composite ceramics, J. Eur. Ceram. Soc. 34 (2014) 1297-1302. doi:10.1016/j.jeurceramsoc.2013.11.034.

[19] S.-M. Kwon, S.-J. Lee, I.-J. Shon, Enhanced properties of nanostructured $\mathrm{ZrO} 2$-graphene composites rapidly sintered via high-frequency induction heating, Ceram. Int. 41 (2015) 835-842.

[20] Z.E. Yin, H. Zhang, Y.R. Kang, J.M. Feng, Y.L. Li, Fabrication of Graphene/Zirconia Nanocomposite by Mixing Graphite Oxide and Zirconia Nanopowders and Pressureless Sintering, in: Key Eng. Mater., Trans Tech Publ, 2012: pp. 65-68.

[21] S. Ramesh, M.M. Khan, H.C.A. Chee, Y.H. Wong, P. Ganesan, M.G. Kutty, U. Sutharsini, W.J.K. Chew, A. Niakan, Sintering behaviour and properties of graphene oxidedoped Y-TZP ceramics, Ceram. Int. 42 (2016) 17620-17625. 
[22] S. Rani, M. Kumar, S. Sharma, D. Kumar, Role of graphene in structural transformation of zirconium oxide, J. Sol-Gel Sci. Technol. 71 (2014) 470-476.

[23] F. Chen, D. Jin, K. Tyeb, B. Wang, Y.-H. Han, S. Kim, J.M. Schoenung, Q. Shen, L. Zhang, Field assisted sintering of graphene reinforced zirconia ceramics, Ceram. Int. 41 (2015) 6113-6116.

[24] K. Markandan, J.K. Chin, M.T.T. Tan, Enhancing Electroconductivity of Yytria-Stabilised Zirconia Ceramic Using Graphene Platlets, in: Key Eng. Mater., Trans Tech Publ, 2016: pp. 1-5.

[25] C. Ramirez, M. Isabel Osendi, Characterization of graphene nanoplatelets-Si3N4 composites by Raman spectroscopy, J. Eur. Ceram. Soc. 33 (2013) 471-477. doi:10.1016/j.jeurceramsoc.2012.09.014

[26] M. Michalkova, M. Kasiarova, P. Tatarko, J. Dusza, P. Sajgalik, Effect of homogenization treatment on the fracture behaviour of silicon nitride/graphene nanoplatelets composites, J. Eur. Ceram. Soc. 34 (2014) 3291-3299. doi:10.1016/j.jeurceramsoc.2014.03.023.

[27] R.K. Chintapalli, F.G. Marro, B. Milsom, M. Reece, M. Anglada, Processing and characterization of high-density zirconia-carbon nanotube composites, Mater. Sci. Eng. A. 549 (2012) 50-59.

[28] H. Rietveld, A profile refinement method for nuclear and magnetic structures, J. Appl. Crystallogr. 2 (1969) 65-71

[29] A.X.S. Bruker, TOPAS V3: General profile and structure analysis software for powder diffraction data, User's Manual, Bruker AXS, Karlsruhe, Ger. (2005).

[30] L.G. Cancado, K. Takai, T. Enoki, M. Endo, Y.A. Kim, H. Mizusaki, N.L. Speziali, A. Jorio, M.A. Pimenta, Measuring the degree of stacking order in graphite by Raman spectroscopy, Carbon N. Y. 46 (2008) 272-275

[31] A.C. Ferrari, J.C. Meyer, V. Scardaci, C. Casiraghi, M. Lazzeri, F. Mauri, S. Piscanec, D. Jiang, K.S. Novoselov, S. Roth, A.K. Geim, Raman spectrum of graphene and graphene layers, Phys. Rev. Lett. 97 (2006).

[32] L.M. Malard, M.A. Pimenta, G. Dresselhaus, M.S. Dresselhaus, Raman spectroscopy in graphene, Phys. Rep. 473 (2009) 51-87. doi:10.1016/j.physrep.2009.02.003.

[33] A.C. Ferrari, Raman spectroscopy of graphene and graphite: Disorder, electron-phonon coupling, doping and nonadiabatic effects, Solid State Commun. 143 (2007) 47-57. doi:10.1016/j.ssc.2007.03.052.

[34] F. Inam, T. Vo, B.R. Bhat, Structural stability studies of graphene in sintered ceramic nanocomposites, Ceram. Int. 40 (2014) 16227-16233. doi:10.1016/j.ceramint.2014.07.058.

[35] C. Casiraghi, A. Hartschuh, H. Qian, S. Piscanec, C. Georgi, A. Fasoli, K.S. Novoselov, D.M. Basko, A.C. Ferrari, Raman spectroscopy of graphene edges, Nano Lett. 9 (2009) 14331441.

[36] B. Román-Manso, E. Domingues, F.M. Figueiredo, M. Belmonte, P. Miranzo, Enhanced electrical conductivity of silicon carbide ceramics by addition of graphene nanoplatelets, J. Eur. Ceram. Soc. 35 (2015) 2723-2731. doi:10.1016/j.jeurceramsoc.2015.03.044
[37] C. Ramirez, L. Garzon, P. Miranzo, M.I. Osendi, C. Ocal, Electrical conductivity maps in graphene nanoplatelet/silicon nitride composites using conducting scanning force microscopy, Carbon N. Y. 49 (2011) 3873-3880. doi:10.1016/j.carbon.2011.05.025.

[38] C. Ramirez, F.M. Figueiredo, P. Miranzo, P. Poza, M. Isabel Osendi, Graphene nanoplatelet/silicon nitride composites with high electrical conductivity, Carbon N. Y. 50 (2012) 36073615. doi:10.1016/j.carbon.2012.03.031.

[39] O. Tapasztó, L. Tapasztó, H. Lemmel, V. Puchy, J. Dusza, C. Balázsi, K. Balázsi, High orientation degree of graphene nanoplatelets in silicon nitride composites prepared by spark plasma sintering, Ceram. Int. 42 (2016) 1002-1006.

[40] R. Poyato, A. Gallardo-López, F. Gutiérrez-Mora, A Morales-Rodríguez, A. Muñoz, A. Domínguez-Rodríguez, Effect of high SWNT content on the room temperature mechanical properties of fully dense 3YTZP/SWNT composites, J. Eur. Ceram. Soc. 34 (2014) 1571-1579. doi:http://dx.doi.org/10.1016/j.jeurceramsoc.2013.12.024.

[41] I. Ahmad, M. Islam, H.S. Abdo, T. Subhani, K.A. Khalil, A.A. Almajid, B. Yazdani, Y. Zhu, Toughening mechanisms and mechanical properties of graphene nanosheet-reinforced alumina, Mater. Des. 88 (2015) 1234-1243.

[42] J. Liu, H. Yan, M.J. Reece, K. Jiang, Toughening of zirconia/alumina composites by the addition of graphene platelets, J. Eur. Ceram. Soc. 32 (2012) 4185-4193. doi:http://dx.doi.org/10.1016/j.jeurceramsoc.2012.07.007.

[43] D.-T. Vu, Y.-H. Han, F. Chen, D. Jin, J.M. Schoenung, D.-Y. Lee, Graphene Nano-Platelets Reinforced $\mathrm{ZrO} 2$ Consolidated by Spark Plasma Sintering, Sci. Adv. Mater. 8 (2016) 312 317.

[44] A. Nieto, D. Lahiri, A. Agarwal, Synthesis and properties of bulk graphene nanoplatelets consolidated by spark plasma sintering, Carbon N. Y. 50 (2012) 4068-4077. doi:10.1016/j.carbon.2012.04.054

[45] A. Gallardo-López, R. Poyato, A. Morales-Rodríguez, A. Fernández-Serrano, A. Muñoz, A. Domínguez-Rodríguez, Hardness and flexural strength of single-walled carbon nanotube/alumina composites, J. Mater. Sci. 49 (2014) 71167123. doi:10.1007/s10853-014-8419-5.

[46] R. Poyato, J. Macías-Delgado, A. García-Valenzuela, Á Gallardo-López, A. Morales-Rodríguez, A. Muñoz, A. Domínguez-Rodríguez, Mechanical and electrical properties of low SWNT content 3 YTZP composites, J. Eur. Ceram. Soc. 35 (2015) 2351-2359. doi:10.1016/j.jeurceramsoc.2015.02.022.

[47] C. Rudolf, B. Boesl, A. Agarwal, In situ indentation behavior of bulk multi-layer graphene flakes with respect to orientation, Carbon N. Y. 94 (2015) 872-878.

[48] A.R. Golkarian, M. Jabbarzadeh, The density effect of van der Waals forces on the elastic modules in graphite layers, Comput. Mater. Sci. 74 (2013) 138-142.

[49] C. Rudolf, B. Boesl, A. Agarwal, In situ indentation behavior of bulk multi-layer graphene flakes with respect to orientation, Carbon N. Y. 94 (2015) 872-878.

[50] P. Miranzo, C. Ramirez, B. Roman-Manso, L. Garzon, H.R. Gutierrez, M. Terrones, C. Ocal, M. Isabel Osendi, M. 
Belmonte, In situ processing of electrically conducting graphene/SiC nanocomposites, J. Eur. Ceram. Soc. 33 (2013) 1665-1674. doi:10.1016/j.jeurceramsoc.2013.01.021.

[51] Y. Fan, L. Wang, J. Li, J. Li, S. Sun, F. Chen, L. Chen, W. Jiang, Preparation and electrical properties of graphene nanosheet/Al2O3 composites, Carbon N. Y. 48 (2010) 1743 1749. doi:http://dx.doi.org/10.1016/j.carbon.2010.01.017.

[52] C. Ramirez, F.M. Figueiredo, P. Miranzo, P. Poza, M.I.

Osendi, Graphene nanoplatelet/silicon nitride composites with high electrical conductivity, Carbon N. Y. 50 (2012) 36073615. doi:10.1016/j.carbon.2012.03.031.

[53] B. Jouault, B. Jabakhanji, N. Camara, W. Desrat, A. Tiberj, J.R. Huntzinger, C. Consejo, A. Caboni, P. Godignon, Y. Kopelevich, Probing the electrical anisotropy of multilayer graphene on the Si face of 6 H-SiC, Phys. Rev. B. 82 (2010) 85438 . 